\title{
DIMENSIONAL ANALYSIS: OVERVIEW AND APPLICATIONS TO PROBLEMS OF SOIL-STRUCTURE INTERACTION
}

\author{
A NON-PEER-REVIEWED PREPRINT UPLOADED TO ENGRXIV
}

\author{
Danilo S. Kusanovic \\ Mechanical and Civil Engineering \\ California Institute of Technology \\ California, CA 91125 \\ dkusanov@caltech.edu
}

\author{
Joaquin Garcia-Suarez \\ Graduate Aerospace Laboratories \\ California Institute of Technology \\ California, CA 91125 \\ ajgarcia@caltech.edu
}

\author{
Domniki Asimaki \\ Mechanical and Civil Engineering \\ California Institute of Technology \\ California, CA 91125 \\ asimaki@caltech.edu
}

November 9, 2019

\begin{abstract}
Dimensional Analysis is a long-established tool widely used in many branches of engineering and science. However, applications in geotechnical engineering, and in particular soil-structure interaction (SSI), have barely been explored, in spite of the method's potential to clarify parameter influence and shed light on the range of response regimes. The purpose of this text is twofold: (a) it intends to provide a brief introduction to Dimensional Analysis specifically tailored to geotechnical engineers by carefully choosing illustrative examples, (b) it uses Dimensional Analysis to study the parameter space in soil-building interaction problems, emphasizing modeling choices and using a finite-element model to demonstrate the concept of physical similarity. The suitability of using certain dimensionless parameters over others is discussed based on and their magnitude and sensitivity analysis.
\end{abstract}

Keywords Dimensional Analysis · Soil-Structure Interaction · Mathematical Modelling

\section{Introduction}

Dimensional Analysis provides a formal procedure to assess the influence of the parameters of a system on its response, regardless of whether or not the equation that connects the parameters is known. Its fundamental underpinning is that laws of nature are independent of the frame of reference or the system of units in which one chooses to express them. Dimensional analysis is widely applied for the design of experiments in several research fields (see, e.g., [1, 2, 3]) because it can provide insight on the behavior of systems that require many parameters to be described, and it can in turn reduce the parameter space required to tune the system response by identifying how physical quantities relate to each other. The dimensionless response comes given as a function of dimensionless groups of parameters. Such groups are obtained through the celebrated Buckingham's theorem [4], which also provides the formal framework to identify new dimensionless parameters to replace others. There is a vast literature on Dimensional Analysis and its engineering ans scientific applications, ranging textbooks from mid and early twentieth century [4] to nowadays [5]. For Spanish-language readers, the authors would further like to recommend the didactic and concise introduction in [6], sixth chapter.

Despite its potential to help understanding and predicting the response of multiparameter systems, its utilization in the fields of geotechnical earthquake engineering and soil dynamics has been limited . Quoting Kausel [7]: 
An additional important lesson to be learned in Soil Dynamics - a lesson which is well known in other areas of science and engineering - is that dimensional analysis can be of invaluable help in establishing the form of some physical phenomena, but it cannot guarantee that such formulas will be physically meaningful.

As exceptions to Kausel's quote, we should mention the experimentalists in the field [8], the perceptive argument pursued by Gibson in his Rankine Lecture [9] and a recent paper by Conti and collaborators [10], where Dimensional Analysis was used to judiciously simplify the parameters controlling motion filtering by a massless foundation. Regarding the latter, the present text may be used as an introduction to better understand the manner in which these researchers applied Dimensional Analysis.

\section{Dimensional Analysis and Buckingham's Theorem}

In mathematical terms, a physically-meaningful relation between response of a system, termed $u$ for the purpose of this discussion, and parameters and variables can be expressed as a dimensionally homogeneous equation,

$$
u=f(\hat{\mathbf{x}} ; \hat{\mathbf{q}}) \text {, with } \hat{\mathbf{q}}=\left(q_{1}, q_{2}, \ldots q_{n}\right)^{\top} \text {, and } \hat{\mathbf{x}}=\left(x_{1}, x_{2}, \ldots x_{d}\right)^{\top} .
$$

The vector $\hat{\mathbf{x}} \in \mathbb{R}^{d}$ represents the independent variables of the system (traditionally, in physical systems, these would be three spatial coordinates and time), $\hat{\mathbf{q}} \in \mathbb{R}^{n}$ represents the $n$-physical parameters, and $f: \mathbb{R}^{d} \times \mathbb{R}^{n} \rightarrow \mathbb{R}$ represents a function whose structure will depend on the nature of the physical phenomenon and the number $\mathrm{k}$ of physical dimensions. For instance, a kinematic problem can be expressed in dimensions of distance and time $(\mathrm{k}=2)$, a dynamical problem adds mass $(\mathrm{k}=3)$, and, a thermomechanical problem adds also temperature $(\mathrm{k}=4)$. Adding other physical quantities, as for instance electric charge, would further increase $k$. Since the aim of Dimensional Analysis is discerning the role of the physical parameters, one may remove $\hat{\mathbf{x}}$ henceforth.

\subsection{The concept of dimensional basis}

At this point, we shall introduce the concept of dimensional basis: such basis is a set of $\mathrm{k}$ parameters (e.g. k elements in $\hat{\mathbf{q}}$ ) whose physical units are independent from each other and can span the physical units of all the parameters of the problem - that is, any physical unit in the problem can be uniquely expressed as a combination (product) of the units of the parameters in the base. Any physical unit can be hence generated through combinations of elements of the basis, and for each out-of-basis parameter and variable one can obtain a combination of basis elements having the same physical unit, what is referred as its "characteristic value" (given the basis choice). Note that this characteristic value may not be unique, in which case reasoning grounded on physical intuition may guide the decision of what value is the most appropiate one in each case. Section 2.2 further elaborates on this point. The ratio between a parameter and its characteristic value yields a dimensionless (or nondimensional) group (of parameters), whereas the ratio between a variable and its characteristic value is likewise termed dimensionless variable.

\subsubsection{Similarity or self-similarity?}

When there are not enough parameters to define the dimensional basis, the problem may lack a characteristic value for one independent variable. Such scenario is equivalent to the case where the characteristic value of some independent variable cannot be defined. In these situations, one resorts to self-similar solutions. This issue appears in idealized situations usually restricted to considerations of intermediate asymptotics of some kind, as for instance it is the case in the inertial sub-range of freely-decaying grid turbulence [11] or Blasius' boundary layer theory [12], often considered in mathematical physics and fluid mechanics, but such will never be the case for the type of problems being considered herein, as the number of parameters shall be greater than $\mathrm{k}=3$.

Moreover, the two possible types of self-similar solutions (first-kind and second-kind, depending on the nature of the similarity variable) must not be confused with two totally different concepts: geometric and physical similarity (also referred as similitude). This nomenclature, aimed to stress the distinct nature of the two concepts, was introduced around the 1950s [12], and it has been used as such ever since. More information on self-similarity and its connection to the Theory of Renormalization Groups (Theoretical Physics) and Lie Groups (Mathematics) can be found in [13] and [14]. The concept of similarity proper is referred in more detail in Section 2.3.

\subsection{System's dimensionless response}

Without loss of generality, it is assumed that the k elements of the basis correspond to the first entries of the vector, for which $q_{i}$ is the $i$-th parameter that does not belong to the basis, and $q_{1}, \cdots, q_{\mathrm{k}}$ are the basis parameters. Returning to Equation (1), assume that the left-hand side is divided by a characteristic value, $u_{c h}=u_{c h}\left(q_{1}, \ldots, q_{\mathrm{k}}\right)$, yielding a nondimensional value, $U$. As this member is dimensionless, so must be the right-hand side. Since the three elements of 
the basis do not form nondimensional groups, the parameter space for this new equation will comprise $m=n-\mathrm{k}$ dimensionless parameters. In mathematical terms this implies that:

$$
U=\frac{u}{u_{c h}}=F(\hat{\boldsymbol{\pi}}), \text { with } \hat{\boldsymbol{\pi}}=\left(\Pi_{1}, \Pi_{2}, \ldots, \Pi_{m}\right)^{\top},
$$

where $\hat{\boldsymbol{\pi}} \in \mathbb{R}^{m}$ is the vector of the $m$-dimensionless parameters constructed from the $\hat{\mathbf{q}} \in \mathbb{R}^{n}$ vector of $n$-physical variables by solving $m=n-\mathrm{k}$ dimensionless equations from a set of $\mathrm{k}$ base units. In this regard, the so-called $\Pi$ groups can be expressed by solving the following equation,

$$
\Pi_{i}=\frac{\left(q_{i}\right)^{a_{i}}}{\left(q_{1}\right)^{a_{2}} \cdots\left(q_{k}\right)^{a_{\mathrm{k}}}} .
$$

The exponents $a_{i}, a_{1}, \cdots a_{k}$ are set to ensure that the final result is dimensionless, thus, the exponents are either integer or rational numbers.

We should also point out here that adding a linearly dependent element to the vector, that is an element defined as a combination of the original ones, it does not change the linear space that the vector spans. Symbolically,

$$
\operatorname{span}\left(\Pi_{1}, \ldots, \Pi_{m}\right)=\operatorname{span}\left(\Pi_{1}, \ldots, \Pi_{m}, \Pi_{m+1}\right),
$$

for $\Pi_{m+1}$ being a combination (product) of the other groups. Therefore, the new element can replace one of the $m$ original elements of the basis and the span remains unaltered, as long as the new element does depend on the element it replaces. In more detail: suppose that the $i$-th element $\Pi_{i}$ is defined as a product of $\Pi_{m+1}$, then we obtain

$$
\operatorname{span}\left(\Pi_{1}, \ldots, \Pi_{i-1}, \Pi_{i}, \Pi_{i+1}, \ldots, \Pi_{m}\right)=\operatorname{span}\left(\Pi_{1}, \ldots, \Pi_{i-1}, \Pi_{m+1}, \Pi_{i+1}, \ldots, \Pi_{m}\right) .
$$

This property allows certain flexibility to consider nondimensional groups.

\subsection{Geometrical and physical similarity}

One last remark regarding the general formulation of Dimensional Analysis. If two systems differ in terms of the values of vector $\hat{\mathbf{q}}$, but yield identical vectors of nondimensional parameters $\hat{\boldsymbol{\pi}}$, then their corresponding responses in nondimensional form, $U$, will also be identical. In those cases it is said that these two systems are physically similar (in other words: despite corresponding to different realizations of Equation (1), Equation (2) is the same for both systems).

This is the baseline principle guiding the proper design of scaled models for experiments (e.g. wind tunnel tests for aircrafts, centrifuge tests of buried structures). This conclusion follows from the moment that is assumed that there is an underlying mathematical model describing the physical phenomenon. This similarity characteristic does not require verification (other than for didactic purposes, as we will pursue later on) and should not come as a surprise for the researcher that employs Dimensional Analysis. For instance, similarity properties derived from Dimensional Analysis can be used for debugging numerical codes, however, numerical codes, working with physical units, should not be used to verify similarity results among models displaying physical similarity.

\section{Some Illustrative Examples}

\subsection{Dynamics without Newton's Laws}

Consider the standard second-order differential equation describing the dynamic behavior of a viscous single-degree-offreedom system [15]:

$$
m \frac{d^{2} u}{d t^{2}}+c \frac{d u}{d t}+k u=F(t)=F_{0} e^{i \varpi t},
$$

where $u$ is displacement (response/dependent variable), $t$ is time (independent variable), $m$ is the corresponding mass, $c$ is the coefficient of viscous damping, $k$ is the corresponding stiffness, and $F(t)$ is a time-varying external harmonic load defined by its amplitude $F_{0}$ and its frequency $\varpi$. This equation corresponds to Newton's Second Law applied to this system. This equation represents the balance of the external load by inertial, viscous and elastic forces, therefore both sides must have the units of force (i.e. $M L T^{-2}$, e.g. newtons, Dyne, poundal). One may as well divide this equation by $m$, expressing it in the so-called canonical form

$$
\frac{d^{2} u}{d t^{2}}+2 \xi \omega_{n} \frac{d u}{d t}+\omega_{n}^{2} u=\frac{F_{0}}{m} e^{i \varpi t}
$$


where $\omega_{n}=\sqrt{k / m}$ is the natural frequency of the system and $\xi=c / 2 / \sqrt{k m}$ is the so-called percentage of critical damping (which happens to be dimensionless). Note that the load introduces a characteristic time-scale, namely the inverse of the frequency $1 / \varpi$. Normally one is interested in analyzing changes across time spans that produce meaningful changes in the load, as meaningful changes in the response will also happen across the same intervals. Let us introduce a dimensionless variable, $\tau$ that measures time in terms of the number of characteristic time intervals, hence $\tau:=\frac{t}{1 / \varpi}=\varpi t$. For instance, $\tau=2 \pi$ correspond to the duration of a complete period of the load. If this dimensionless variable is introduced in Equation (7), we obtain:

Now, divide by $\omega_{n}^{2}$ to yield:

$$
\varpi^{2} \frac{d^{2} u}{d \tau^{2}}+2 \xi \omega_{n} \omega \frac{d u}{d \tau}+\omega_{n}^{2} u=\frac{F_{0}}{m} e^{i \tau},
$$

$$
\left(\frac{\varpi}{\omega_{n}}\right)^{2} \frac{d^{2} u}{d \tau^{2}}+2 \xi\left(\frac{\varpi}{\omega_{n}}\right) \frac{d u}{d \tau}+u=\frac{F_{0}}{k} e^{i \tau} .
$$

Clearly, as can be seen by the last term of the left-hand side $(u)$, the last equation has units of displacements. Since the right-hand side also must have units of displacements, the amplitude $F_{0} / k$ represents a certain characteristic displacement. Incidentally, one can recognize that, had dynamic behavior been absent, the displacement amplitude would have been just the quasi-static $u_{s t}:=F_{0} / k$. In the same spirit that $\tau$ was introduced to quantify time in terms of a characteristic value, a new variable $\tilde{u}:=u / u_{s t}$ can be introduced to compare the displacement to the non-dynamic displacement. To make this new variable appear, just divide Equation (8b) by $u_{s t}$ :

$$
\left(\frac{\varpi}{\omega_{n}}\right)^{2} \frac{d^{2} \tilde{u}}{d \tau^{2}}+2 \xi\left(\frac{\varpi}{\omega_{n}}\right) \frac{d \tilde{u}}{d \tau}+\tilde{u}=e^{i \tau} .
$$

The latter is truly a non-dimensional equation, involving no physical units. Of course, the physics of the problem are still there, encapsulated in the definition of $\tilde{u}, \tau$ and within the dimensionless parameters that appeared along the process, i.e., $\varpi / \omega_{n}=\varpi / \sqrt{k / m}$ and $\xi=c / 2 / \sqrt{k m}$. No information has been lost during the process, as only trivial algebraic manipulations were involved. In light of Equation (9), one can state that Newton's Second Law suggests that $\varpi / \omega_{n}$ and $\xi$ are the two parameters controlling the response, at least when expressed in terms of $\tilde{u}$.

However, one may ask: could one attain the same conclusion without relying on Newton'a laws? To answer this question, let us suppose that without knowing $F=m a$ or D'Alembert's Principle, one may surmise that $m, c, k$ are the system parameters that control the response. On this basis alone, on may posit the existence of a functional relation:

$$
u=f\left(m, c, k, F_{0}, \varpi\right),
$$

relating the value of the response to the value of the parameters of the problem. Dividing this expression by $u_{s t}$, the left-hand side becomes non-dimensional $(\tilde{u})$ and so must the right-hand side as well, to preserve dimensional homogeneity:

$$
\tilde{u}=\tilde{f}\left(m, c, k, F_{0}, \varpi\right)
$$

A logical concern can be raised regarding the function $\tilde{f}$ : if it has to yield a dimensionless result, how will it do so if the parameters that appear within it have physical units? The key lies in acknowledging that the dependence of $\tilde{f}$ on the parameters must happen through dimensionless combinations of groups of them. For example, recall that the parameters that appear in the denominator of $\tau, \xi$ and $\varpi / \omega_{n}$ were $F_{0}, m, k$. Let us use them to form dimensionless groups, e.g., use them to furnish a dimensional basis. The only possible way of writing the arguments of $\tilde{f}$ as dimensionless groups using these parameters is:

$$
\tilde{u}=\tilde{f}\left(1, \frac{c}{\sqrt{k m}}, 1,1, \frac{\varpi}{\sqrt{k / m}}\right) .
$$

Acknowledging that the two parameters have been introduced before:

$$
\tilde{u}=\tilde{f}\left(2 \xi, 1,1, \frac{\varpi}{\omega_{n}}\right)=\tilde{f}\left(\xi, \frac{\varpi}{\omega_{n}}\right) .
$$

The following conclusion is reached: the response of the system, when expressed in terms of $\tilde{u}$, must be a function of just two dimensionless groups of physical parameters: $\xi$ and $\varpi / \omega_{n}$. The same conclusion was attained by studying Equation (6), but this time there has been no direct appeal to Newton's second law. Finally, note that this application of Dimensional Analysis reveals the controlling parameters of the response, yet it does not yield the functional form of $\tilde{f}$. 


\subsection{A cautionary tale: long-wavelength thrust on retaining walls resting on bedrock}

Consider the model introduced by [16] in the context of studying seismic earth pressures on rigid retaining walls. The material is assumed to be linear-elastic, with material constants $\mu$ (shear modulus) and $\nu$ (Poisson's ratio). The model features a semi-infinite layer of soil, height $H$, restrained at the base $(y=0)$ by rigid bedrock and at one of the sides $(x=0)$ by a rigid smooth wall. The horizontal body force $\gamma$, is used as a proxy for the forced base shaking. In the framework of quasi-static (or long-wavelength) analysis, the frequency content of the load can be disregarded to focus only on the amplitude. Figure 1 shows a simplified layout of the retaining wall's configuration.

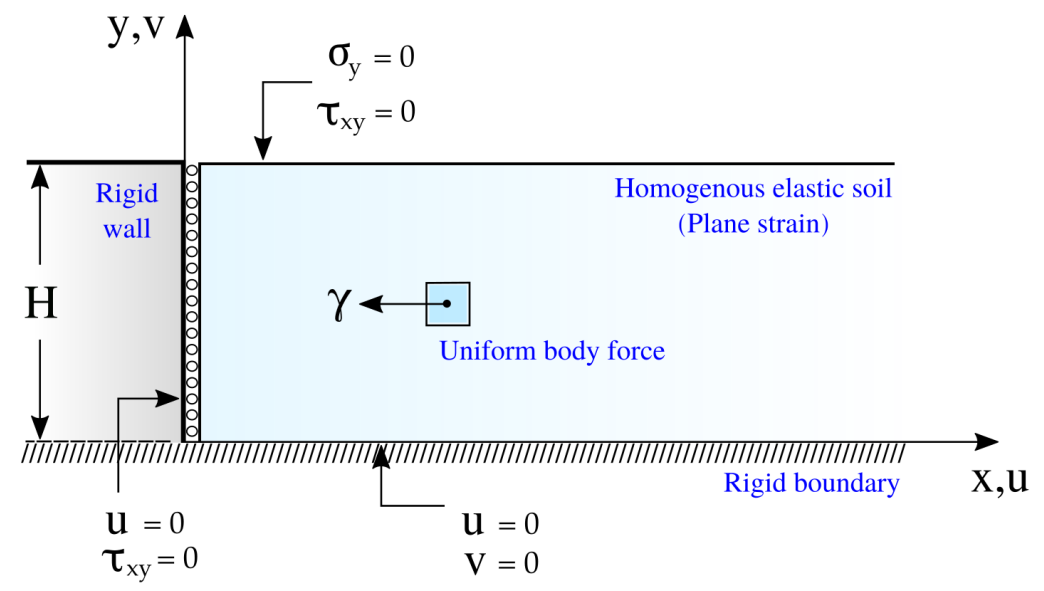

Figure 1: Scheme of the system to be considered (modificied from [17])

Let us begin by assuming that the earth thrust on the wall, $Q_{b}$, is described by a specific functional that must depend on the (independent) parameters of the problem:

$$
Q_{b}=q(\nu, \mu, \rho, \gamma, H),
$$

for which a possible dimensional basis could be $\{\rho, \gamma, H\}$. This dimensional basis introduces a characteristic length $H$, a characteristic time $\sqrt{H / \gamma}$ and a characteristic mass $\rho H^{3}$. The thrust has units of stress times length (as it is the integral of stresses over the wall), or of force per unit length, thus it can be assigned a characteristic value using the variables in the basis, as outlined by Equation (3),

$$
\left[Q_{b}\right]=M L T^{-2} L^{-1}=M T^{-2} \rightarrow\left(\rho H^{3}\right)(\sqrt{H / \gamma})^{-2}=\rho \gamma H^{2} .
$$

To complete the non-dimensionalization process, a characteristic value must be assigned to $\mu$ :

$$
[\mu]=M L T^{-2} L^{-2}=M L^{-1} T^{-2} \rightarrow\left(\rho H^{3}\right)\left(H^{-1}\right)(\sqrt{H / \gamma})^{-2}=\rho \gamma H .
$$

Under this base, Equation (13) in non-dimensional form becomes:

$$
\frac{Q_{b}}{\rho \gamma H^{2}}=\tilde{q}\left(\nu, \frac{\mu}{\rho \gamma H}\right)=\tilde{q}\left(\nu, \frac{\ell}{H}\right)
$$

where $\ell=\mu / \rho \gamma=V_{s}^{2} / \gamma$ represents a characteristic length of the problem, and $V_{s}$ is the shear wave velocity of the soil.

Given realistic values of $V_{s}$ for most soils, and realistic values of $\gamma$ for most earthquakes, $\ell$ will be of the order of hundreds or thousands of meters (for instance, if $V_{s}=100 \mathrm{~m} / \mathrm{s}$ and $\gamma=0.1 \mathrm{~g}=1 \mathrm{~m} / \mathrm{s}^{2}$, then $\ell=10^{4} \mathrm{~m}$, that is ten kilometers); at the same time, for typical retaining walls, $H$ will be of the order of magnitude of few meters. Thus, for the scope of this study, $\ell / H \gg 1$; the inverse of this ratio can be interpreted as a characteristic strain of the problem (more on the reasons for this interpretation in the application example in Section 4). Note that this small-strain assumption is implicit in linear theory. Then, assuming linear elasticity, the long wavelength approximation corresponds to the asymptotic limit $\ell / H \rightarrow \infty$, therefore

$$
\frac{Q_{b}}{\rho \gamma H^{2}}=\tilde{q}\left(\nu, \frac{\ell}{H} \rightarrow \infty\right)=\tilde{q}_{\mathrm{LE}}(\nu),
$$


where the subscript LE stands for Linear Elasticity. Under these assumptions, the non-dimensional thrust $\tilde{q}$ will depend solely on the Poisson's ratio $\nu$, that is on the compressibility of the soil.

Let us next analyze the result we would obtain, had we used a different basis, for example $\{\rho, \gamma, \mu\}$. Characteristic values are not so easily identified for this new base, so we resort to Equation (3). $\Pi_{1}=\nu$ does not change, and $\Pi_{2}$ is in this case:

$$
\Pi_{2}=\frac{H}{\rho^{a} \gamma^{b} \mu^{c}}
$$

The denominator has the following units:

$$
\left[\rho^{a} \gamma^{b} \mu^{c}\right]=\left(M L^{-3}\right)^{a}\left(L T^{-2}\right)^{b}\left(M L^{-1} T^{-2}\right)^{c}=M^{a+c} L^{-3 a+b-c} T^{-2 b-2 c} .
$$

Since $a, b, c$ must be such that the final dimension is length, $a+c=0,-3 a+b-c=1$, and $-2 b-2 c=0$. The solution of this system of equations is $a=b=-c=-1$, thus:

$$
\Pi_{2}=\frac{H}{\rho^{-1} \gamma^{-1} \mu}=\frac{H}{\ell},
$$

where $\ell$ is the characteristic length-scale mentioned above. Let us perform one more exercise and find a characteristic value of $Q_{b}$ based on the parameters of the base: since $Q_{b} /\left(\rho^{a} \gamma^{b} \mu^{c}\right)$ is dimensionless, $a, b, c$ must be such that the final dimension of the denominator is force per unit length, that is $M T^{-2}$. Therefore $a+c=1$ and $-3 a+b-c=0$, and the solution of this system of equations is $a=b=-1$, and $c=2$. Thus the characteristic value of the thrust is $\mu^{2} / \rho \gamma=\mu \ell$. In conclusion, the counterpart of Equation (16) for the second base is:

$$
\frac{Q_{b}}{\mu \ell}=\bar{q}\left(\nu, \frac{H}{\ell}\right) .
$$

By applying the same long wavelength argument that was used with Equation (16), one could (erroneously) think that

$$
\frac{Q_{b}}{\mu \ell}=\bar{q}\left(\nu, \frac{H}{\ell} \rightarrow 0\right)=\bar{q}_{\mathrm{EL}}(\nu) .
$$

For the sake of sheer consistency, Equation (16) and Equation (21) must verify

$$
Q_{b}=\mu \ell \bar{q}_{\mathrm{LE}}(\nu)=\rho \gamma H^{2} \tilde{q}_{\mathrm{LE}}(\nu) \rightarrow \bar{q}_{\mathrm{LE}}(\nu)=\frac{\rho \gamma H^{2}}{\mu \ell} \tilde{q}_{\mathrm{LE}}(\nu)=\left(\frac{H}{\ell}\right)^{2} \tilde{q}_{\mathrm{LE}}(\nu) .
$$

Clearly a contradiction is reached: if $\bar{q}_{\mathrm{LE}}(\nu)$ scales as $(H / \ell)^{2}$, it cannot at the same time depend on that dimensionless group. Where is the error? The issue is in the limit in Equation (21): namely, it is assumed that the limit corresponds to reaching an asymptote, when instead, it should have been assumed that the function displays a certain scaling in the parameter as $H / \ell \rightarrow 0$. Inspired by Equation (22), let us correct Equation (21) by assuming a quadratic scaling of the parameter $(H / \ell)$ as the limit is approached:

$$
Q_{b}=\mu \ell \bar{q}\left(\nu, \frac{H}{\ell} \rightarrow 0\right)=\mu \ell\left(\frac{H}{\ell}\right)^{2} \tilde{q}_{\mathrm{LE}}(\nu)=\frac{\mu H^{2}}{\ell} \tilde{q}_{\mathrm{LE}}(\nu)=\rho \gamma H^{2} \tilde{q}_{\mathrm{LE}}(\nu) .
$$

This time, there is complete agreement between the new equation and Equation (16).

Could we have foreseen the issues that arose when taking the limit in Equation (13)? On one hand,we should have noticed that the very large characteristic value assigned to the thrust in Equation (20) defies any realistic value for the problem in hand. On the other hand, a subtler argument could have been explored: linear elasticity presupposes a linear relation between external forces and displacement field, and by extension, a linear relation between external forces and stress field; as the thrust is the integral of certain stresses over the height of the wall, $H$, this linear relation extends to the relation between external load amplitude and thrust: therefore, the scaling of $Q_{b}$ in terms of $\gamma$ must be linear at the limit $H / \ell \rightarrow 0$. This condition explicitly prohibits Equation (21), which would entail a scaling proportional to $\gamma^{-1}$.

This example has illustrated that applying the Buckingham Theory does not automatically yield correct scaling laws: the researcher must exercise common sense and caution, in particular, when defining characteristic values of response. The ensuing example deepens on this aspect. 


\subsection{On the importance of characteristic values}

Recall Equation (6) corresponding to the SDOF introduced in the first example, this time with no damping. Instead of a harmonic load, consider now a certain bell-shaped load evolution:

$$
m \ddot{u}+k u=p_{0}\left(\frac{t}{t_{0}}\right) e^{-t / t_{0}},
$$

$t_{0}$ is evidently the characteristic time scale introduced by the load in this case. Assume $\tau=t / t_{0}$ and $\tilde{u}=u / \mathcal{U}$ (the characteristic value of the displacement, $\mathcal{U}$, is to be defined). Use these nondimensional values to find the corresponding dimensionless form of Equation (24)

$$
\frac{\partial \tilde{u}}{\partial \tau^{2}}+\left(t_{0} \omega_{n}\right)^{2} \tilde{u}=\frac{p_{0} t_{0}^{2}}{m \mathcal{U}} \tau e^{-\tau} .
$$

Define $r=t_{0} \omega_{n}$ and $\mathcal{U}$ such that $p_{0} t_{0}^{2} / m \mathcal{U}=1 \rightarrow \mathcal{U}=p_{0} t_{0}^{2} / m$. Thus:

$$
\frac{\partial \tilde{u}}{\partial \tau^{2}}+r^{2} \tilde{u}=\tau e^{-\tau}
$$

Now, imagine that $r \gg 1$ holds for a sub-class of systems. One may ask if this circumstance introduces some potential simplification on the system. In order to verify it, one can analyze the scaling of each term with respect to the parameter $r$ :

$$
\underbrace{\frac{\partial \tilde{u}}{\partial \tau^{2}}}_{\mathcal{O}(1)}+\underbrace{r^{2} \tilde{u}}_{\mathcal{O}\left(r^{2}\right) \gg 1}=\underbrace{\tau e^{-\tau}}_{\mathcal{O}(1)},
$$

provided that $r \gg 1$, the $r^{2} \tilde{u}$ term is expected to be much larger than the other two, which a priori do not scale with $r$. As there are no other terms in the equations, one may think that to satisfy the equilibrium, the only possibility is that $\tilde{u} \approx 0$ vanishes, so that the other two terms of similar magnitude can balance each other. However, if this were the case, the system would behave as a rigid body, what seems unlikely, based on common sense and physical intuition. The problem arose from the non-dimensionalization, namely, from the choice of characteristic value. The actual characteristic displacement must be much smaller than the characteristic displacement that was assumed, so let us return to Equation (25), and redefine it as:

$$
\frac{\partial \tilde{u}}{\partial \tau^{2}}+r^{2} \tilde{u}=\frac{p_{0} t_{0}^{2}}{m \mathcal{U}} \tau e^{-\tau}
$$

divide by $r^{2}$

$$
\underbrace{\frac{1}{r^{2}} \frac{\partial \tilde{u}}{\partial \tau^{2}}}_{\ll 1}+\tilde{u}=\underbrace{\frac{p_{0} t_{0}^{2}}{m r^{2} \mathcal{U}}}_{1} \tau e^{-\tau},
$$

thus, in this case $\mathcal{U}=p_{0} /\left(t_{0}^{2} / m r^{2}\right)=p_{0} t_{0}^{2} /\left(m t_{0}^{2} k / m\right)=p_{0} / k=u_{s t}$. Hence, under this assumption, the logical characteristic displacement has to be the static displacement. Recall that $r=t_{0} \omega_{n} \sim t_{0} / T_{n}$, which means that $r \gg 1$ is equivalent to $t_{0} \gg T_{n}$ : in other words, the characteristic time of the load is large relative to the natural period of the system, which means that the load as seen by the system changes very slowly (and thus there is plenty of time for the to adapt to any small perturbation in load amplitude). Since the system experiences slow changes, accelerations -and thus inertial forces- balance the external load at each time step, and the system's response is equal to the static displacement corresponding to the load level at that same time step.

This example has illustrated that the characteristic values must be argued carefully, and can change for different regimes of the system, which themselves are defined on the basis of the system's dimensionless groups.

\subsection{A final note}

While examples in this section may have illustrated the challenges associated with Dimensional Analysis and nondimensionalization, we should underscore the added value in bringing forth these considerations: not only deep physical insight into the problem is provided, also the physical interpretation of the dimensionless groups and the non-dimensionalization enable the application of perturbation and asymptotic methods [18], some of the most important mathematical tools available for the analysis of complex engineering problems in different response regimes. 


\section{Dimensional Analysis Applied to Soil-Structure Interaction}

The remainder of the paper focuses on a problem of soil-structure-interaction (SSI) under seismic loading. The problem involves a building (characterized by its first modal modal mass, height, and fundamental period); a rectangular shallow foundation (characterized by its homogeneous density and by two linear-elastic isotropic constants); the underlying soil half-space (characterized by its density and by two linear-elastic isotropic constants); and the seismic loading characteristics (represented in our case by a Ricker pulse [19] of a given dominant frequency and dominant acceleration amplitude). Perfect bonding between soil and foundation is assumed. We are interested in understanding how structure and soil parameters affect the structural response, given a seismic excitation. A list of the physical parameters $q_{i}$ that govern this phenomenon, as well as their physical dimensions, are provided in Table 1.

\begin{tabular}{cc|l}
\hline \multicolumn{2}{c|}{ Parameters } & \\
\hline$T_{b}$ & $T$ & Fixed-base fundamental period of the building. \\
$h_{b}$ & $L$ & First-modal height of the fixed-base building. \\
$B$ & $L$ & Half-width of the building foundation. \\
$D$ & $L$ & Depth of the building foundation. \\
$m_{b}$ & $M$ & First-modal mass of the fixed-base building. \\
$\rho_{f}$ & $M L^{-3}$ & Foundation density. \\
$\nu_{f}$ & - & Poisson's ratio of foundation material. \\
$\rho_{s}$ & $M L^{-3}$ & Soil density. \\
$\nu_{s}$ & - & Poisson's ratio of the soil. \\
$V_{s}$ & $L T^{-1}$ & Shear-wave velocity of the soil. \\
$V_{f}$ & $L T^{-1}$ & Shear-wave velocity within the foundation. \\
$a_{p}$ & $L T T^{-2}$ & Amplitude of the acceleration ground motion. \\
$T_{p}$ & $T$ & Ground motion period with the highest energy. \\
\hline \hline
\end{tabular}

Table 1: The $n$-physical parameters of the SSI problem to be considered in the dimensional analysis.

The response of the structure (represented by $u$, not necessarily a displacement) in this idealized SSI context can be written in terms of the physical parameters $\hat{\mathbf{q}}$ listed in Table 1 as,

$$
u=f\left(T_{b}, h_{b}, B, D, m_{b}, \rho_{f}, \nu_{f}, \rho_{s}, \nu_{s}, V_{s}, V_{f}, a_{p}, T_{p}\right) .
$$

Based on Equation (29), the number of variables for the SSI problem are thirteen $(n=13)$, whereas the number of independent units for this case are three $(\mathrm{k}=3)$, i.e., time $T$, length $L$, and mass $M$. Thus, the number of dimensionless groups that can be found is ten $(m=10)$. In spite of the reduction granted by considering nondimensional groups, dealing at once with the complete parameter space, spanned by ten independent parameters, is virtually impossible. to reduce the parameter space, we must first determine the role of each parameter group, and argue that, for real scenarios, the value of some groups can be neglected.

Before proceeding, we first detail the interpretation and treatment of each nondimensional group. To do so, let us analyze the relevance of each subsystem independently.

\subsection{Subsystem constituents of the SSI problem}

\subsubsection{Wave propagation within the soil}

We have chosen to approximate the seismic shaking by a vertically propagating, in-plane shear wave characterized by a narrowband pulse referred to as a Ricket pulse [19]. The angle of incidence, measured with respect to the normal to the surface, is fixed and assumed $\theta=0$. The wave propagation is defined in terms of the velocity field as:

$$
\dot{u}_{g}(y, t)=\dot{u}_{p}\left(1-\left(\frac{t-y / V_{s}}{T_{p}}\right)^{2}\right) e^{-\frac{1}{2}\left(\frac{t-y / V_{s}}{T_{p}}\right)^{2}} .
$$

The wave profile is controlled by a characteristic wave velocity $\dot{u}_{p}$, and a characteristic time $T_{p}$. The corresponding characteristic wavelength is $\lambda_{s}=V_{s} T_{p}$. The stress induced by the propagating wave is:

$$
\tau_{x y}=\rho_{s} V_{s} \dot{u}_{g}(y, t),
$$

thus a characteristic value (order of magnitude) of stress in the soil is:

$$
\left|\tau_{x y}\right| \sim \rho_{s} V_{s} \dot{u}_{p}
$$




\subsubsection{Foundation embedded in soil}

The foundation in the soil halfspace acts like a scatterer, modifying the incident wavefield. Thus, the foundation can be conceptualized as an inclusion at the interface between building and bedrock. Elastic wave theory [20] shows that the amplitude of the scattered wavefield depends on the characteristic length of the inclusion (in this case, the width of the foundation base) over the the characteristic wavelength of the excitation $\left(B / \lambda_{s}\right)$.

For a given $B$, if the foundation response is similar to the response in the far-field, $B / \lambda_{s} \ll 1$, which indicates that this would be the case if the foundation were much smaller than the characteristic wavelength of the incident wave. On the other hand, if the response is very different from the far field, $B / \lambda_{s} \sim 1$, which would be the case if the foundation were of the same order of magnitude as the characteristic wavelength: this scenario represents substantial kinematic interaction.

\subsubsection{Superstructure}

Since our interest is to understand the global response of the structure, we next minimize the number of free parameters by focusing on undamped buildings whose response are dominated by their first mode. Only radiation damping will be considered in the ensuing as mechanism of energy loss.

The superstructure reacts to the movement of the base either immediately or with a certain delay. If the natural period of the structure is much larger than that of the excitation $\left(T_{b} \gg T_{p}\right)$, then the foundation-building system will respond as a rigid body subjected to base motion. On the other hand, if $T_{b} \sim T_{p}$, the building will deform relative to the base shortly after the seismic wavefront reaches the foundation. In both cases, since the center of mass of the foundation-building system is well above the ground, the inertia forces in the building will induce an overturning moment at the foundation level. The building base rocking in turn has to be accommodated by the soil adjacent to the foundation, a compatibility condition that yields additional soil displacement that further disrupts the coherent incident wavefield. This phenomenon is referred as inertial interaction. We should also note here that for the purpose of this study, we ignore gravity and the associated geometric nonlinearities it entails (known as $P-\delta$ effects. Since we only limit our analysis to the linear regime, the effect of gravity can be summed to the response later on.

The movement of the building-foundation system deforms the surrounding soil in turn, hence the system is shedding waves into the soil half-space. The energy these outward-propagating waves carry is effectively an energy loss in the building-foundation system, this it is referred as radiational damping.

\subsection{Application of Dimensional Analysis}

As outlined in Section 2, we must next extract a dimensional basis from the parameters in Table 1. The selected parameters are $B$ (which provides relative length to characterize the geometry), $V_{s}$ (which defines a characteristic time in conjunction with $B$ ), and $\rho_{s}$ (to represent inertia). Once again, this choice is arbitrary inasmuch there are other possible bases that could provide an equally valid dimensional basis.

Without loss of generality, we assume at this point that $u$ represents a displacement. A characteristic displacement is given by the Ricker pulse amplitude $\left(\sim a_{p} T_{p}^{2}\right)$, thus $U=u / a_{p} T_{p}^{2}$ in our case. We then apply the Buckingham $\Pi$-theorem to obtain the $m$-dimensionless parameters associated to the $n$-physical variables by using Equation (3). Equation (29) can thus be rewritten in dimensionless fashion:

$$
\begin{aligned}
U=\frac{u}{a_{p} T_{p}^{2}} & =F\left(\frac{T_{b} V_{s}}{B}, \frac{h_{b}}{B}, 1, \frac{D}{B}, \frac{m_{b}}{\rho_{s} B^{3}}, \frac{\rho_{f}}{\rho_{s}}, \nu_{f}, 1, \nu_{s}, 1, \frac{V_{f}}{V_{s}}, \frac{a_{p} B}{V_{s}^{2}}, \frac{T_{p} V_{s}}{B}\right) \\
& =F\left(\frac{T_{b} V_{s}}{B}, \frac{h_{b}}{B}, \frac{D}{B}, \frac{m_{b}}{\rho_{s} B^{3}}, \frac{\rho_{f}}{\rho_{s}}, \nu_{f}, \nu_{s}, \frac{V_{f}}{V_{s}}, \frac{a_{p} B}{V_{s}^{2}}, \frac{T_{p} V_{s}}{B}\right) \\
& =F\left(\Pi_{1}, \Pi_{2}, \Pi_{3}, \Pi_{4}, \Pi_{5}, \Pi_{6}, \Pi_{7}, \Pi_{8}, \Pi_{9}, \Pi_{10}\right) .
\end{aligned}
$$

Let us next analyze these dimensionless groups, and argue their relative importance:

(1) $\Pi_{1}=\frac{T_{b}}{B / V_{s}}$ represents the ratio between the natural oscillations of the building and the characteristic time of the soil response. More will be said about it in the ensuing discussion but it suffices to mention here that this parameter plays a very important role in the system response.

(2) $\Pi_{2}=\frac{h_{b}}{B}$ is a geometric ratio between the height of the building and the width of its base. It provides a measure of the objective slenderness of the building. $\Pi_{2} \sim 1$ will correspond to low building whereas $\Pi_{2} \sim 10$ or $\Pi_{2} \sim 100$ will correspond to tall or very tall buildings. $\Pi_{2}$ is an important geometric parameter that 
influences the coupling between soil and structural responses, and it can span orders of magnitudes, depending on the typology of building (from one-storey building to tall slender ones). This ratio is arguably very relevant because it affects the rocking of the structure.

(3) $\Pi_{3}=\frac{D}{B}$ provides a measure of the embedment of the foundation in the soil. This is another important geometric parameter that influences the coupling between soil and foundation. For instance, it can take values in a relatively wide interval: from $\Pi_{6}<0.5$, shallow footing-type foundations, to $\Pi_{6} \geq 5$, deep caisson-like foundation.

(4) $\Pi_{4}=\frac{m_{b}}{\rho_{s} B^{3}}$ represents the ratio between mass of a characteristic volume of soil to the mass of the building (for the sake of averting any doubt, it is assumed that all off-plane dimensions characterizing the geometry of the structure are $\sim B$. For two-dimensional problems, this parameter is a ratio between masses per unit length. This ratio relates inertia of the building to inertia of the soil surrounding the structure, and similarly to $\Pi_{4}$, this parameter is potentially important.

(5) $\Pi_{5}=\frac{\rho_{f}}{\rho_{s}}$ represents the ratio between the density of the foundation versus the one of the soil. This ratio will in general be $\sim 1$ and will influence the inertial interaction.

(6) $\Pi_{6}=\nu_{f}$ is the Poisson ratio of the foundation, which is restrained to be between zero and one half; the magnitude variability hints limited importance when compared to the other groups, the exception being the incompressibility limit, as when $\nu_{s} \rightarrow 1 / 2$ there is a related parameter, the bulk stiffness, that goes asymptotically to infinity. Hence, except in the prior limit, once the value is fixed, admissible perturbations in its value should not significantly affect the response.

(7) $\Pi_{7}=\nu_{s}$ is the Poisson's ratio of the soil. Similar arguments as those used for $\nu_{f}$ are compelled herein. It is also implicitly assumed that the response cannot scale dramatically rapidly with $\Pi_{7}$, thus the small variations in the value of $\Pi_{7}$ that are permitted cannot yield large changes in the response.

(8) $\Pi_{8}=\frac{V_{f}}{V_{s}}$ represents the ratio of the foundation material shear wave velocity to the corresponding value in the soil. For the purpose of this study, it shall be assumed that the waves travel much faster within the foundation (namely, that the foundation is much stiffer but not much denser than the soil), and hence assume $\Pi_{3} \gg 1$. Assuming that the limit reaches an asymptote (recall 3.2), the response must possess a limit as $\Pi_{3} \rightarrow \infty$ (plainly put, there must be a point at which increasing this parameters does not meaningfully affect the output). Restricting our focus to conditions that satisfy such condition, perturbations on its value must not significantly perturb the response (this limit scenario correspond to the case of a "rigid foundation").

(9) $\Pi_{9}=\frac{B}{V_{s}^{2} / a_{p}}$ represents the ratio between a characteristic dimension of the structure to the length scale defined by the ground shaking intensity and the material properties of the soil. Intuition dictates this ratio must relate to the characteristic strain in the system (similarly to what was discussed in section 3.2). For linear elasticity to be valid, $\Pi_{9} \ll 1$; for real case scenarios, intensity, soil material properties and geometry of the structure usually yield $\Pi_{9} \ll 1$ so the influence of this parameter is likely negligible for the class of problems we focus on.

(10) $\Pi_{10}=\frac{T_{p} V_{s}}{B}$ represents the ratio between the characteristic wavelength of the load to a characteristic length of the foundation. Hence this parameter controls the kinematic interaction in the system. It is also accepted that the consequences of this interaction do not take precedence over the inertial interaction in the majority of cases. Accordingly, $\Pi_{10}$ seems not as important as $\Pi_{1}$ and $\Pi_{4}$ to describe the building behavior, yet it remains essential to understand the full system response.

We next employ Equation (5) to redefine the following two groups: $\Pi_{1}^{\prime}=\Pi_{2} / \Pi_{1}=h_{b} / V_{s} T_{b}$, and $\Pi_{4}^{\prime}=\Pi_{4} / \Pi_{2}=$ $m_{b} / \rho_{s} h_{b} B^{2}$. Note that these new parameters coincide with the ones used by Veletsos [21] and Bielak [22]. As argued earlier in Section 2, these parameters are neither "inferior" nor "superior" to others, but convenient inasmuch describing the soil response concerns, and, moreover, happen to be the customary ones in the field.

We have furthermore argued that $\Pi_{6}$ and $\Pi_{7}$ cannot affect the structural response much and without loss of generality, we heretofore consider $\Pi_{8}=0.2$ and $\Pi_{5}=0.35$. Additionally, we take the limit of $\Pi_{8} \rightarrow \infty$ (rigid foundation) and $\Pi_{9} \rightarrow 0$ (linear-elastic response). Thus four parameters can be discounted from the parameter space accordingly, in 
turn, Equation (32) can be simplified as follows:

$$
\begin{aligned}
U & =F\left(\Pi_{1}, \Pi_{2}, \Pi_{3}, \Pi_{4}, \Pi_{5}, \Pi_{6}=0.20, \Pi_{7}=0.35, \Pi_{8} \rightarrow \infty, \Pi_{9} \rightarrow 0, \Pi_{10}\right) \\
& =F\left(\Pi_{1}, \Pi_{2}, \Pi_{3}, \Pi_{4}, \Pi_{5}, \Pi_{10}\right) \\
& =F\left(\Pi_{2} / \Pi_{1}, \Pi_{2}, \Pi_{3}, \Pi_{4} / \Pi_{2}, \Pi_{5}, \Pi_{10}\right) \\
& =F\left(\Pi_{1}^{\prime}, \Pi_{2}, \Pi_{3}, \Pi_{4}^{\prime}, \Pi_{5}, \Pi_{10}\right) .
\end{aligned}
$$

We could further reduce the parameter space from six to three using additional assumptions $\left(\Pi_{1}^{\prime}, \Pi_{2}\right.$ and $\left.\Pi_{3}\right)$. These assumptions will be discussed later, and the three most sensitive parameters to SSI problems will be defined, which coincide with the parameters highlighted in $[21,22]$.

\subsection{Illustrative examples and demonstration of physical similarity}

In this part, we consider the $\Pi$ theorem to devise physically-similar computer simulations and test that their responses are indeed similar, in the sense of Equation (2).
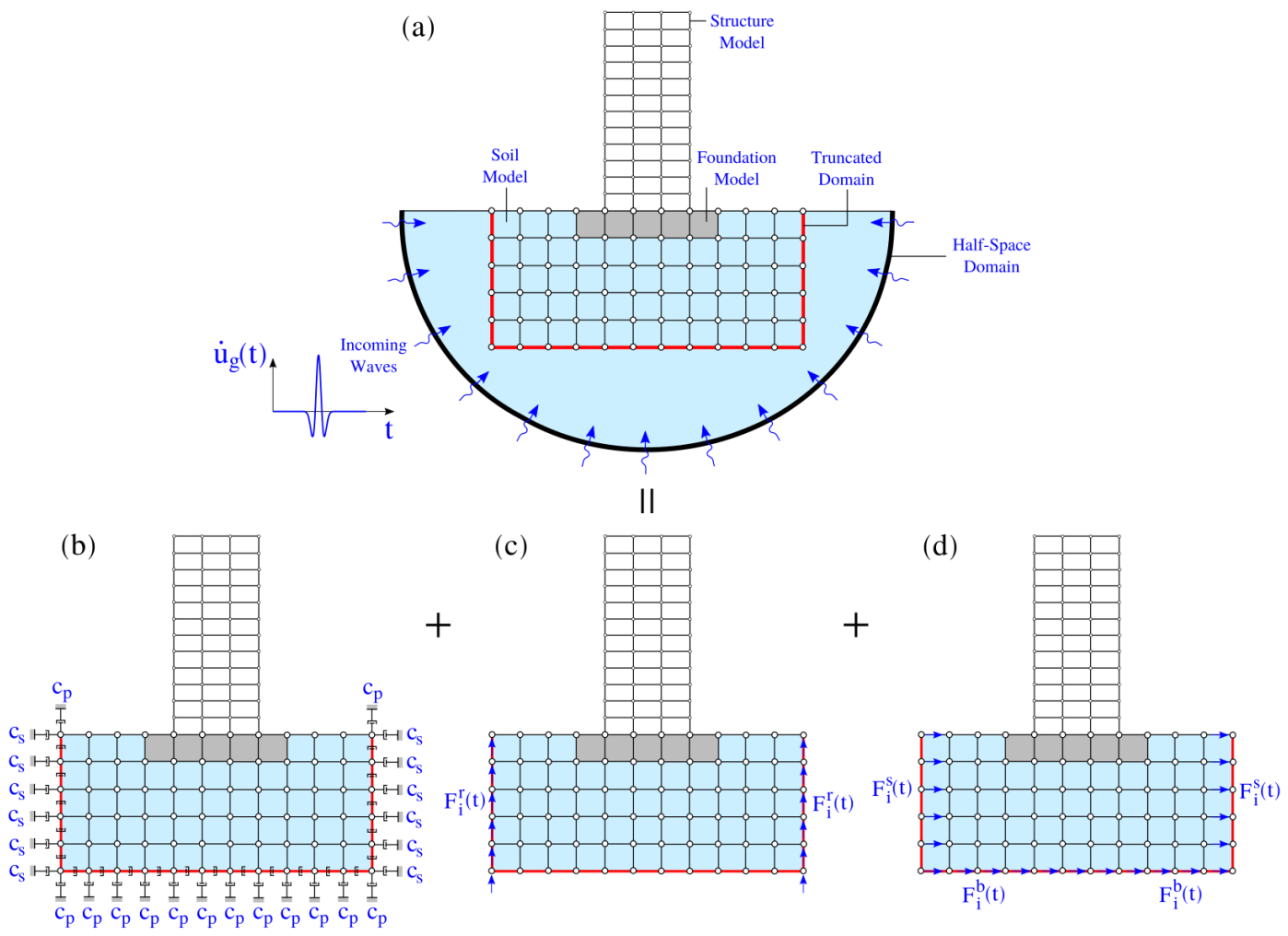

Figure 2: Reduced infinite half-space model using absorbing dashpots elements.

We use the finite element method [23, 24] (also known as direct modeling method [25, 26] in the SSI community) to compute the building structure response on elastic half-space - see Figure 2a. We specifically truncate the semi-infinite half-space along the red line in Figure 2a, and introduce Lysmer dashpots accompanied by prescribed nodal forces to artificially approximate an interface transparent to both incoming and outgoing waves [27, 28]. The Lysmer dashpot coefficient shown in Figure 2b for absorbing outgoing waves are computed as:

$$
\begin{aligned}
& c_{s}=\rho_{s} V_{s} t_{h} \Delta x_{i}, \\
& c_{p}=\rho_{s} V_{p} t_{h} \Delta z_{i} .
\end{aligned}
$$

and the corresponding nodal forces, shown in Figure $2 \mathrm{c}$, that translate the vertically propagating incoming shear waves within the domain of interest are

$$
\begin{aligned}
& F_{i}^{s}=c_{p} \dot{u}_{i}(t), \\
& F_{i}^{b}=c_{s} \dot{u}_{g}(t) .
\end{aligned}
$$


In Equation (34) and (35) the variable $t_{h}=1.0[\mathrm{~m}]$ is the out-of-plane thickness of the truncated soil domain; $\Delta x_{i}$ and $\Delta z_{i}$ represent the tributary $i$-th length which are different for corner and inner boundary nodes; $V_{s}$ is the shear wave velocity of the soil; and $V_{p}$ is the compressive wave velocity in the soil. It is worth to point out that the boundary conditions enforced in this manner can almost perfectly absorb body waves with angles of incidence greater than $30^{\circ}$ degrees with respect to the vertical axis, and will lose their effectiveness for lower angles of incidence or for surface waves. Similarly, in Equation (36) and (37) we use a Ricker-wavelet as an incident plane wave at the base of the model:

$$
\dot{u}_{g}(t)=\left(1-2 \beta\left(t-t_{0}\right)^{2}\right) \exp \left[-\beta\left(t-t_{0}\right)^{2}\right],
$$

where $\beta=\left(\pi f_{0}\right)^{2}, f_{0}$ is the characteristic frequency, $t_{0}$ is the time that corresponds to the peak velocity of the pulse. In all simulations, we consider a characteristic frequency $f_{0}=1 / T_{p}$, and a peak time velocity $t_{0}=1.0[s]$. The node velocity $\dot{u}_{i}(t)$ on the left and right boundary can be computed in closed form as:

$$
\dot{u}_{i}(t)=\dot{u}_{g}\left(-\frac{z_{i}}{V_{s}}+t\right)+\dot{u}_{g}\left(\frac{z_{i}-2 H}{V_{s}}+t\right)
$$

where $z_{i}$ is the vertical coordinate of the $i$-th node, and $H$ is the total vertical hight of the truncated domain. Finally, the reactive forces $F_{i}^{r}$ applied on the left and right boundaries are $F_{i}^{r}=\rho_{s} V_{s} t_{h} \Delta z_{i} \dot{u}_{i}(t)$, which enforce the shear deformation of the truncated domain.

The soil mesh is represented by quadrilateral solid elements with two degrees of freedom per node and dimensions $\Delta x=0.5[\mathrm{~m}], \Delta z=0.5[\mathrm{~m}]$. The soil model has a horizontal length of $350[\mathrm{~m}]$ and a vertical depth of $200[\mathrm{~m}]$ in all simulations. In each case, we analysed the building response for a time window $t$ of dimensionless time $\tilde{t}=V_{s} t / h_{b}=50.0$.

The building mesh is represented by frame structural elements with three degrees of freedom per node, and length $\Delta l=0.5[\mathrm{~m}]$. Model I has a column and beam cross section of $0.65 \times 0.65\left[\mathrm{~m}^{2}\right], 3$ columns and 6 floors. Model V has a column section of $0.925 \times 0.925\left[\mathrm{~m}^{2}\right]$, a beam section of $0.90 \times 0.90\left[\mathrm{~m}^{2}\right], 5$ columns and 19 floors. Finally, Models II, III, and IV all have 5 columns and 13 floors; however the column cross section are $1.16 \times 1.16\left[\mathrm{~m}^{2}\right], 0.75 \times 0.75\left[\mathrm{~m}^{2}\right]$, and $0.595 \times 0.595\left[\mathrm{~m}^{2}\right]$ respectively, while the beam cross section are $1.05 \times 1.05\left[\mathrm{~m}^{2}\right], 0.735 \times 0.735\left[\mathrm{~m}^{2}\right]$, and $0.585 \times 0.585\left[\mathrm{~m}^{2}\right]$. The five different configuration properties are summarized in Table 2 , and depicted schematically in Figure 3.

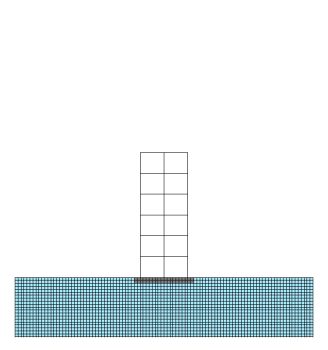

(a) Model I

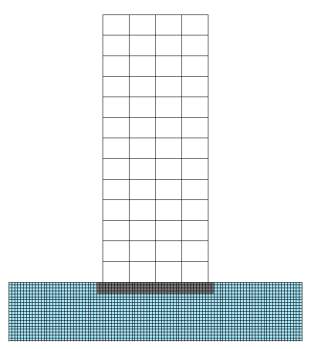

(b) Model III

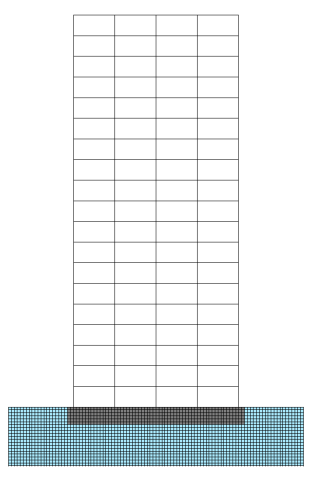

(c) Model V

Figure 3: Representation of the similar soil-foundation-structure models described in Table 2.

Note that models II, III, and IV shown in Figure 3 b have the same building $\left(h_{b}\right)$ and foundation $(B, D)$, but their material properties (as characterized by $\left.T_{b}\right)$ and the material properties of the soil $\left(V_{s}, \rho_{s}\right)$ are modified such that the dimensionless parameters are virtually identical. On the other hand, models I, III, and V shown in Figure 3a-c have different geometry for the building and foundation $\left(h_{b}, B, D\right)$ and different material properties $\left(T_{b}, \rho_{s}, V_{s}\right)$, but once again the dimensionless parameters are the same. In this example, the dimensional parameter vector is set such that $\hat{\boldsymbol{\pi}}=(0.1031,3.0922,0.200,0.200,1.546,0.200,0.350,20.00,0.0001,0.0719)$ for the five configurations. 
A PREPRINT - NOVEMBER 9, 2019

\begin{tabular}{cc|ccccc}
\hline \multicolumn{6}{c|}{ Parameters } & \multicolumn{5}{c}{ Soil-Foundation-Structure Models } \\
Variable & Units & I & II & III & IV & V \\
\hline$T_{b}$ & {$[s]$} & 0.700 & 0.650 & 1.060 & 1.500 & 1.299 \\
$h_{b}$ & {$[\mathrm{~m}]$} & 14.84 & 31.25 & 30.92 & 30.79 & 44.61 \\
$B$ & {$[\mathrm{~m}]$} & 5.0 & 10.0 & 10.0 & 10.0 & 15.0 \\
$D$ & {$[\mathrm{~m}]$} & 1.0 & 2.0 & 2.0 & 2.0 & 3.0 \\
$m_{b}$ & {$[\mathrm{~kg}]$} & $2.40 \cdot 10^{5}$ & $1.75 \cdot 10^{6}$ & $1.06 \cdot 10^{6}$ & $9.94 \cdot 10^{5}$ & $2.80 \cdot 10^{6}$ \\
$\rho_{f}$ & {$\left[\mathrm{~kg} / \mathrm{m}^{3}\right]$} & 2200.0 & 4370.0 & 2908.0 & 2484.3 & 3116.1 \\
$\nu_{f}$ & {$[-]$} & 0.20 & 0.20 & 0.20 & 0.20 & 0.20 \\
$\rho_{s}$ & {$\left[\mathrm{~kg} / \mathrm{m}^{3}\right]$} & 1450.0 & 2796.8 & 1880.8 & 1613.7 & 2397.0 \\
$\nu_{s}$ & {$[-]$} & 0.35 & 0.35 & 0.35 & 0.35 & 0.35 \\
$V_{s}$ & {$[\mathrm{~m} / \mathrm{s}]$} & 215.14 & 480.80 & 282.84 & 199.10 & 346.41 \\
$V_{f}$ & {$[\mathrm{~m} / \mathrm{s}]$} & 4302.86 & 9619.00 & 5656.85 & 3981.89 & 6928.20 \\
$a_{p}$ & {$\left[\mathrm{~m} / \mathrm{s}^{2}\right]$} & 1.158 & 4.590 & 1.000 & 0.498 & 1.000 \\
$T_{p}$ & {$[\mathrm{~s}]$} & 0.323 & 0.227 & 0.4919 & 0.6985 & 0.602 \\
\hline \hline
\end{tabular}

Table 2: Physical variable considered for constructing physically similar soil-structure-interaction models.

Based on the elements used for the basis, the displacement and acceleration responses are normalized as $u / a_{p} T_{p}^{2}$ and $\ddot{u} / a_{p}$ respectively, and they are plotted in Figures 4 to 7 respectively. The horizontal and vertical responses of the five configurations are evaluated at:

1) the leftmost column at the ground level i.e., $\tilde{\Delta}^{g}$,

2) the first modal height i.e., $\tilde{\Delta}^{h}$,

3) the roof i.e., $\tilde{\Delta}^{r}$.

\subsubsection{Physical similarity in display}

In particular, the response of the first set of physically-similar soil-structure-interaction models, i.e., I, III, and V is shown in Figure 4 and 5.
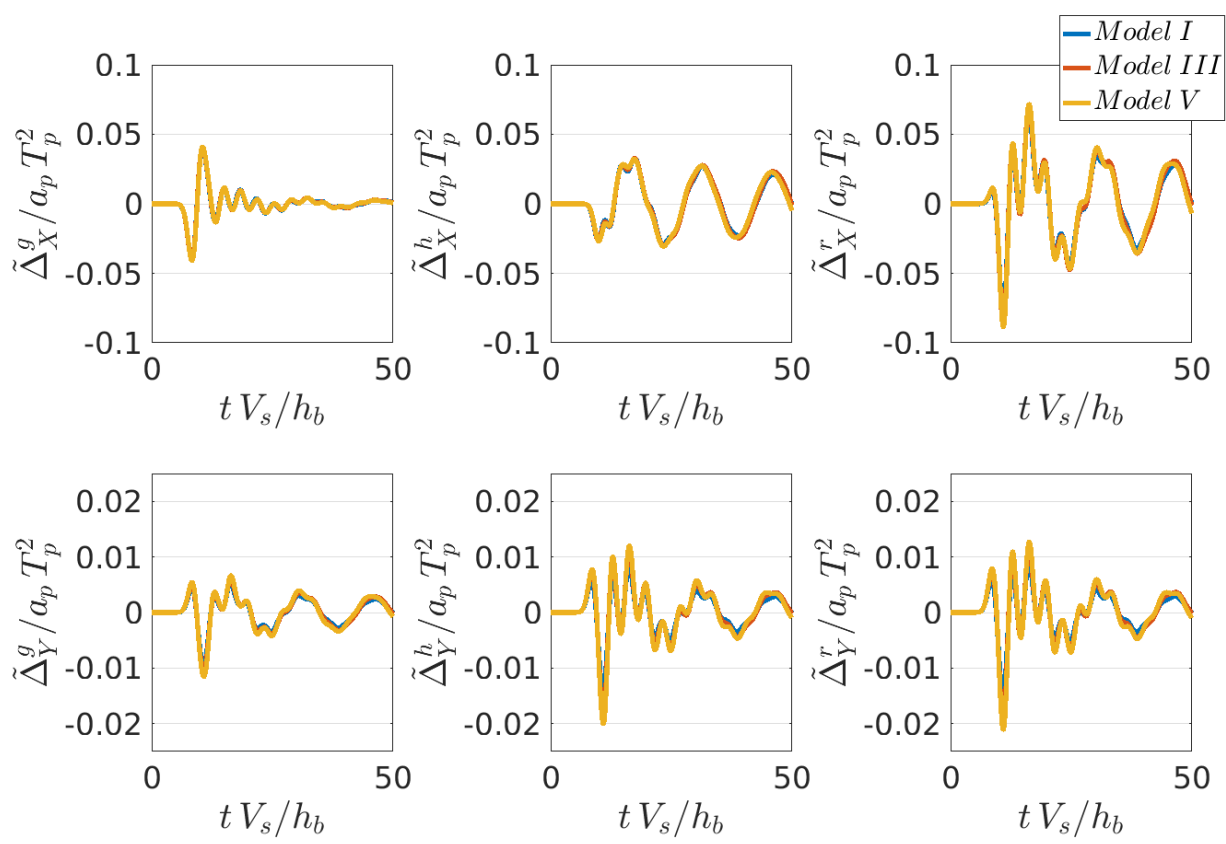

Figure 4: Absolute horizontal (top row) and vertical (bottom row) building's displacement evaluated at the ground level $\tilde{\Delta}^{g}$, first modal high $\tilde{\Delta}^{h}$, and roof $\tilde{\Delta}^{r}$ for configuration I, III, and V. 

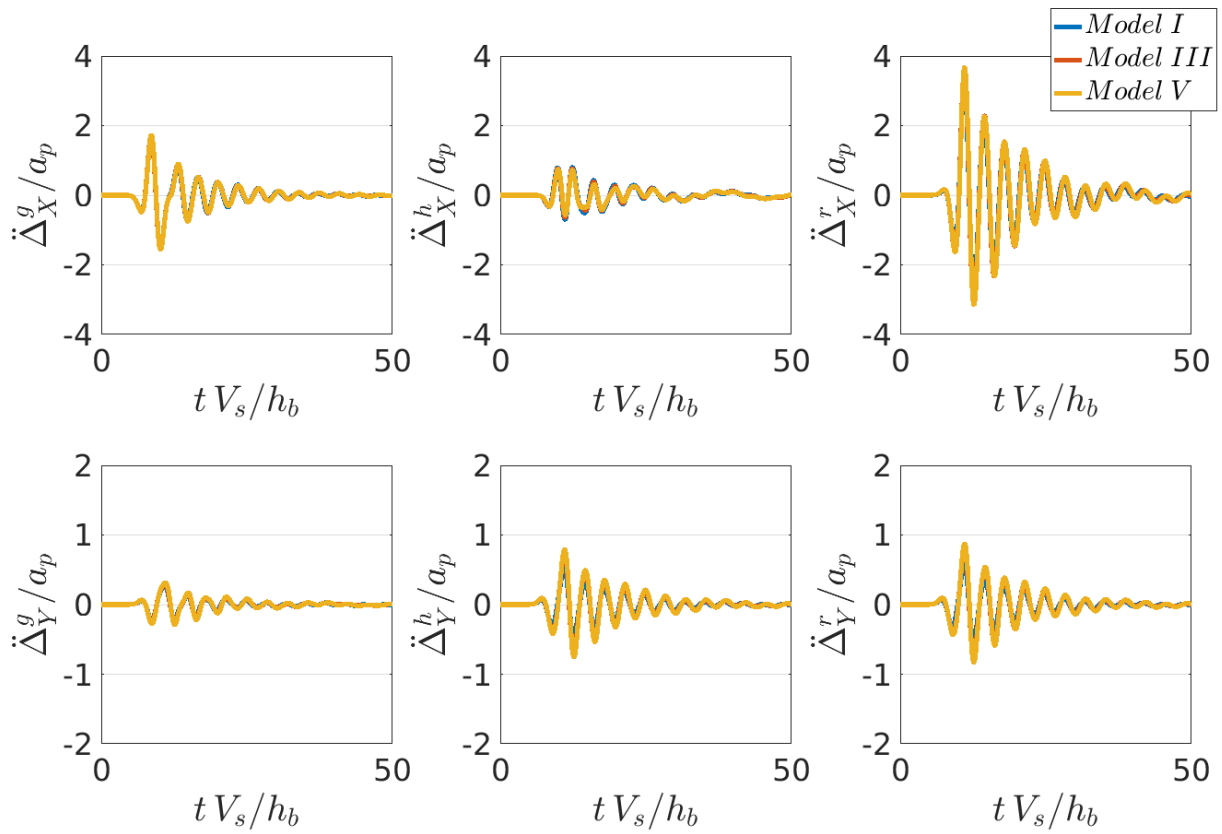

Figure 5: Absolute (top row) and vertical (bottom row) building's acceleration evaluated at the ground level $\ddot{\Delta}^{g}$, first modal high $\ddot{\Delta}^{h}$, and roof $\ddot{\Delta}^{r}$ for configuration I, III, and V.

Here, we confirm that the three normalized response quantities (acceleration and displacement vectors) are almost identical. Similar results were obtained for the SSI models II, III, and IV; their responses are depicted in Figures 6 and 7 respectively.
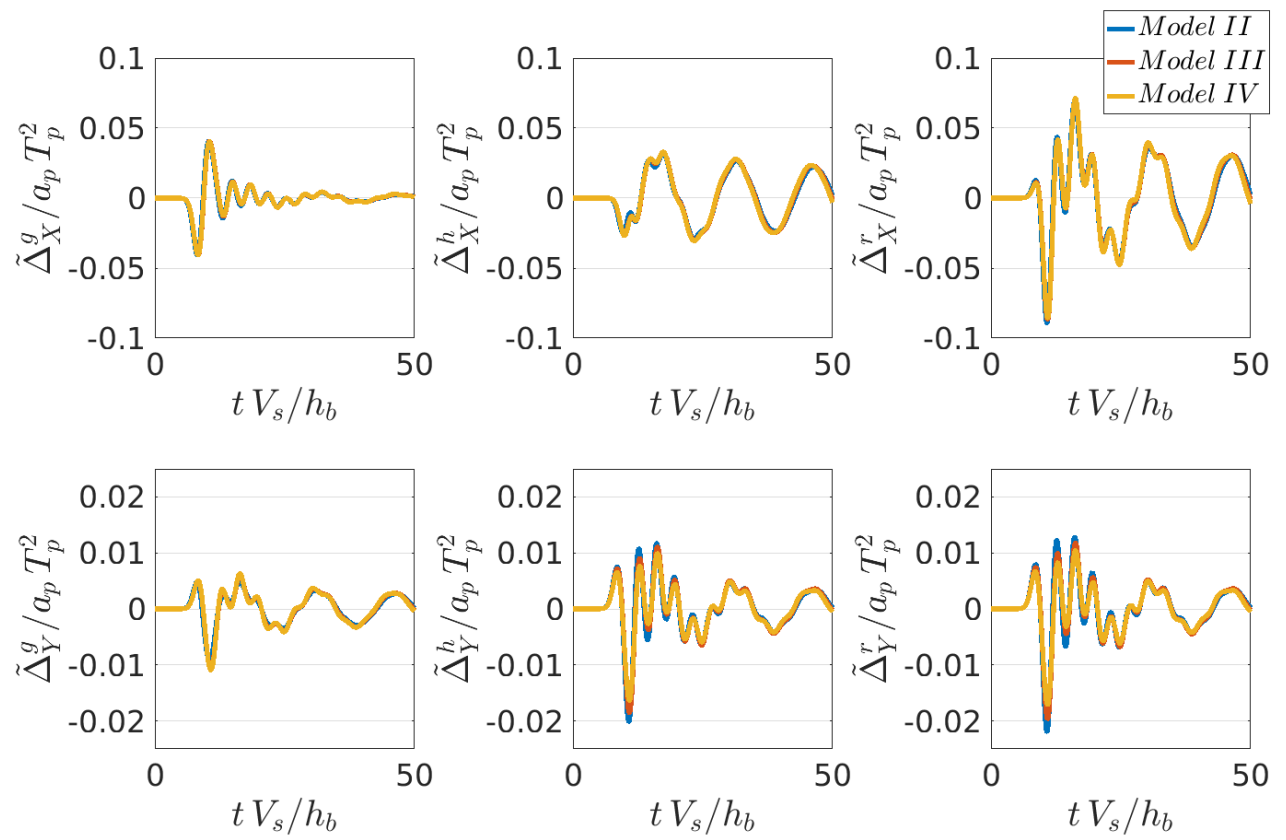

Figure 6: Absolute horizontal and vertical building's displacement evaluated at the ground level $\tilde{\Delta}^{g}$, first modal high $\tilde{\Delta}^{h}$, and roof $\tilde{\Delta}^{r}$ for configuration II, III, and IV. 

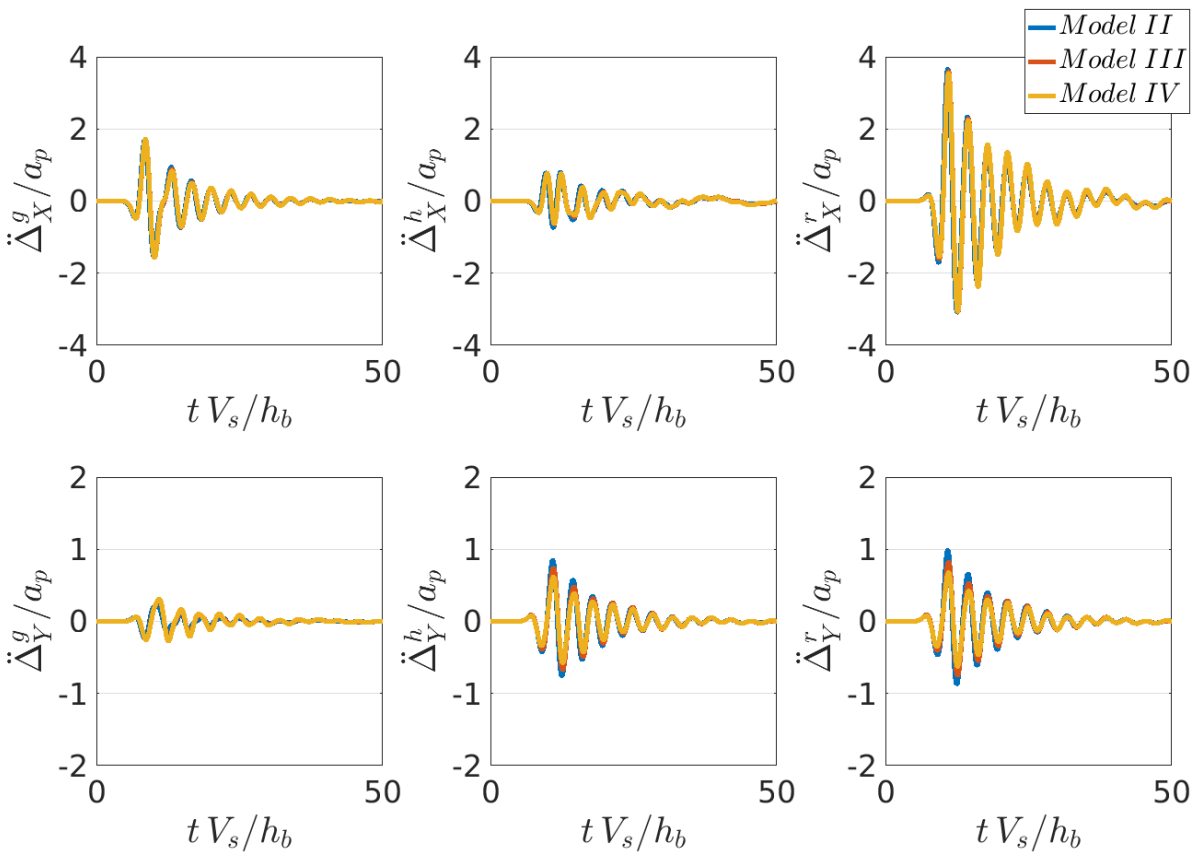

Figure 7: Absolute horizontal and vertical building's acceleration evaluated at the ground level $\ddot{\Delta}^{g}$, first modal high $\ddot{\Delta}^{h}$, and roof $\ddot{\Delta}^{r}$ for configuration II, III, and IV.

In a more detailed analysis, Figure 6 and 7 show small variability of the peak responses for Models II and IV, but the global response is very well captured. The discrepancies are attributed primarily to aspects of the numerical models (e.g. the axial column stiffness) that were not accounted for in the simplified configuration we used for the Dimensional Analysis. For example, the axial column stiffness was not accounted for in the parameter space, which only represented each building by their first modal properties, and thus, scaling parameters such as the cross section of columns and beams were only considered through their influence on the modal parameters, not explicitly. In addition, the evaluation of the response at the first modal height is not obtained exactly at $h_{b}$ but at the closest mesh node. Nevertheless, the responses evaluated at the ground level i.e., surface of the foundation in the horizontal and vertical directions, are identical in both sets of similar structural configurations, as it could not be otherwise by virtue Equation (2).

\subsubsection{Numerical parameter sensitivity analysis}

The previous results show that the responses are identical as long as the non-dimensional parameters are the same, and the simplified model properly resembles the more-complex model used in our simulations. We then proceeded to evaluate the sensitivity of the dimensional parameters associated with the building response. In particular, we numerically quantified the sensitivity as follows:

$$
\frac{\partial U(\hat{\boldsymbol{\pi}} ; \hat{t})}{\partial \Pi_{i}}=\lim _{\Delta \Pi_{i} \rightarrow 0} \frac{U\left(\Pi_{1}, \ldots, \Pi_{i}+\Delta \Pi_{i}, \ldots, \Pi_{m} ; \hat{t}\right)-U(\hat{\boldsymbol{\pi}} ; \hat{t})}{\Delta \Pi_{i}} .
$$

Since the functional form of the responses is unknown, we evaluated Equation (40) using standard finite differences. We first generated a $10 \%$ perturbation around the i-th dimensional parameter $\Pi_{i}$, enforcing that the rest $m-1$ dimensional parameters to stay constant. We then computed the normalized response, and finally computed the sensitivity of each parameter as a function of time as shown in Equation (40). Results of the maximum sensitivity of each parameter in terms of displacement and acceleration at the foundation, first modal height and roof levels are provided in Table 3. 

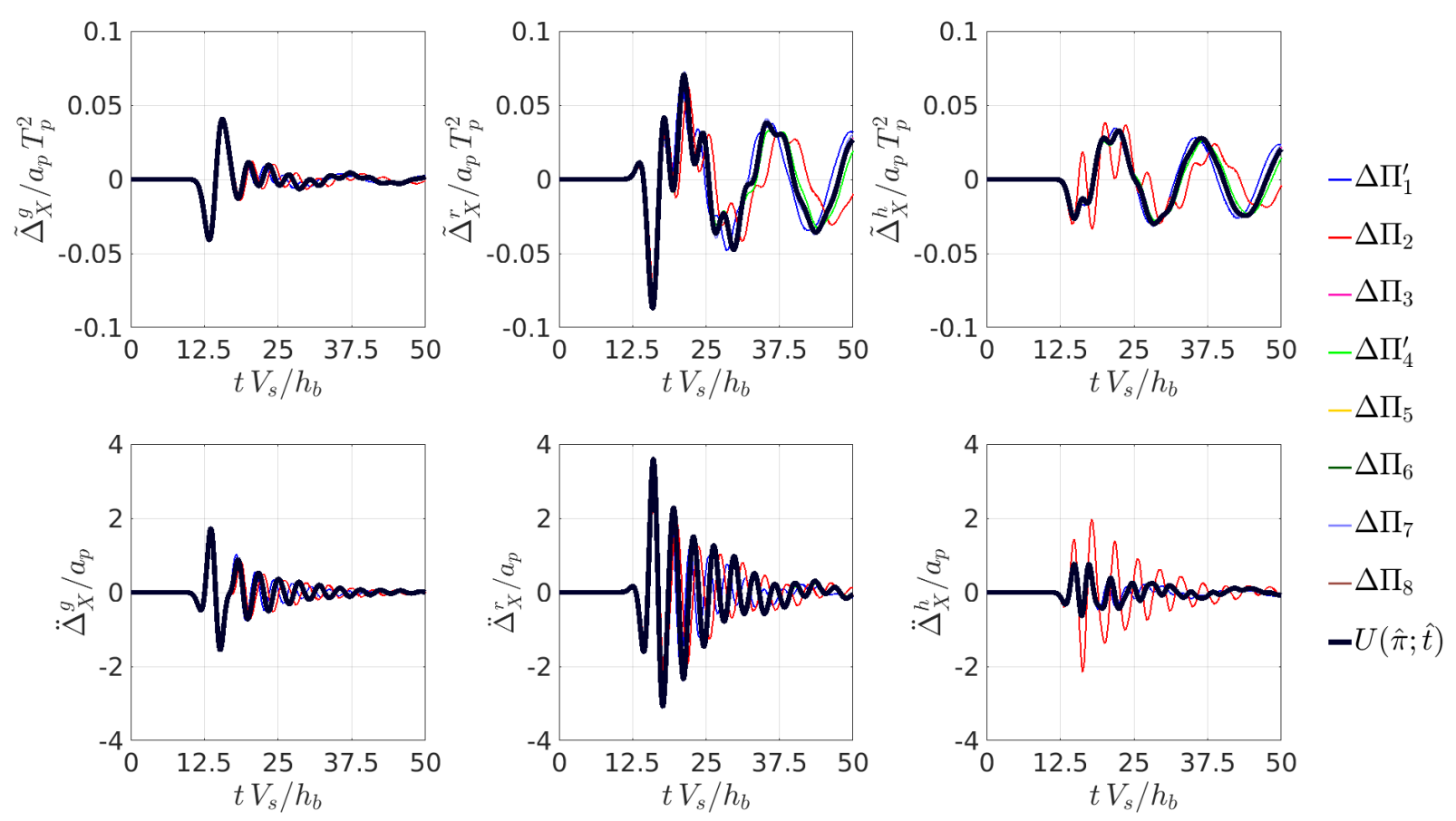

Figure 8: Similar soil-foundation-structure models described in Table 2.

\begin{tabular}{cc|cccccccc}
\hline \multicolumn{2}{c|}{ Dimensionless } & \multicolumn{8}{c}{ Dimensional Parameters } \\
\multicolumn{2}{c}{ Response } & $\Pi_{1}^{\prime}$ & $\Pi_{2}$ & $\Pi_{3}$ & $\Pi_{4}^{\prime}$ & $\Pi_{5}$ & $\Pi_{6}$ & $\Pi_{7}$ & $\Pi_{8}$ \\
\hline \multirow{2}{*}{$u$} & $\tilde{\Delta}_{X}^{g}$ & 0.805 & 0.040 & 0.011 & 0.061 & 0.001 & 0.000 & 0.014 & 0.000 \\
$a_{p} T_{p}^{2}$ & $\tilde{\Delta}_{X}^{h}$ & 2.947 & 0.190 & 0.075 & 0.675 & 0.001 & 0.001 & 0.187 & 0.000 \\
& $\tilde{\Delta}_{X}^{r}$ & 1.289 & 0.135 & 0.078 & 0.513 & 0.000 & 0.000 & 0.135 & 0.000 \\
\hline \multirow{2}{*}{$\ddot{u}$} & $\ddot{\Delta}_{X}^{g}$ & 56.78 & 2.75 & 0.58 & 2.99 & 0.08 & 0.018 & 0.39 & 0.001 \\
$a_{p}$ & $\ddot{\Delta}_{X}^{h}$ & 191.9 & 8.77 & 2.51 & 9.57 & 0.15 & 0.075 & 1.32 & 0.006 \\
& $\ddot{\Delta}_{X}^{r}$ & 41.29 & 6.72 & 0.50 & 2.21 & 0.05 & 0.047 & 0.64 & 0.001 \\
\hline \hline
\end{tabular}

Table 3: Sensitivity analysis results applied to the dimensionless variables for soil-structure-interaction models.

The values presented in Table 3 suggest that the most sensitive parameter, that is the one that controls the normalized response of the structure, is $\Pi_{1}$. Parameters $\Pi_{2}$ and $\Pi_{4}$ also influence strongly the response of the structure. As shown in Figure 8, the three top parameters in descending order of importance are $\Pi_{1}, \Pi_{2}$ and $\Pi_{4}$.

We should highlight here that the non-dimensional parameter $\Pi_{3}$ (relative embedment) was constrained to small values in all simulations, what is why it didn't surface as critically influential. It can be shown, however, that for deeper embedded foundations (e.g. underground parking garages), the sensitivity to this dimensional parameter becomes meaningful, and should be added to the three parameters listed above.

To conclude, compare these last plots and table to the outcome of the reasoning carried out during the discussion of the $\Pi$ groups: the more consequential parameters can be argued out by "force of reason", and simulations confirmed previsions to a large extent. Take this as a testimony of the potential of Dimensional Analysis to aid untangling the inherent intricacies of complex parametric problems in soil structure interaction and beyond.

\section{Conclusions}

Let us summarize our conclusions as follows

1) We have presented a introduction that, despite its brevity, presents the general aspects of Dimensional Analysis as well as strives to clarify some misconceptions (e.g., between self-similarity and similarity).

2) Dimensional Analysis is a critical tool to extract subtle implications of analytical models. 
3) We have assorted a selection of simple examples to illustrate some of the principal operative tests a researcher may encounter while utilizing of this tool.

4) A throughout application of Dimensional Analysis to Soil-Structure Interaction has been presented, including the process for obtaining the dimensionless groups that control the response, and discussions on their physical interpretation and their relative importance.

5) Simulations displaying the significance of physical similarity have been included. These also serve as a token to test the insight derived from Dimensional Analysis, commenting on the limitations of simplified models and a critical assessment of the consequences.

\section{Acknowledgments}

The authors are thankful to Prof. R. Conti (University of Rome) for his suggestions regarding applications of Dimensional Analysis in the geotechnical engineering literature.

The format used in this preprint has been dispensed by Mr. George Kour through Overleaf under Creative Commons CC BY 4.0.

\section{References}

[1] M. Zlokarnik. Dimensional Analysis and Scale-up in Chemical Engineering. Springer Berlin Heidelberg, 2012.

[2] R. Kurth. Dimensional Analysis and Group Theory in Astrophysics. Elsevier Science, 2013.

[3] H.G. Hornung. Dimensional Analysis: Examples of the Use of Symmetry. Dover Books on Physics. Dover Publications, 2013.

[4] Henry Louis Langhaar. Dimensional analysis and theory of models, volume 2. Wiley New York, 1951.

[5] Volker Simon, Bernhard Weigand, and Hassan Gomaa. Dimensional analysis for engineers. Springer, 2017.

[6] Antonio Barrero Ripoll and Miguel Pérez-Saborid Sánchez-Pastor. Fundamentos y Aplicaciones de la Mecánica de Fluidos. McGraw-Hill, 2005.

[7] Eduardo Kausel. On the frequencies of inhomogeneous soil strata: Dobry's paradox. Soil Dynamics and Earthquake Engineering, 47:38-40, 2013.

[8] David Muir Wood. Geotechnical modelling. CRC press, 2014.

[9] RE Gibson. Some results concerning displacements and stresses in a non-homogeneous elastic half-space. Geotechnique, 17(1):58-67, 1967.

[10] Riccardo Conti, Marco Morigi, and Giulia MB Viggiani. Filtering effect induced by rigid massless embedded foundations. Bulletin of Earthquake Engineering, 15(3):1019-1035, 2017.

[11] Peter Davidson. Turbulence: an introduction for scientists and engineers. Oxford University Press, 2015.

[12] Lev D Landau and Evgeny Mikhailovich Lifshitz. Fluid mechanics, volume 6 of. Course of theoretical physics, 4, 1959.

[13] Nigel Goldenfeld. Lectures on phase transitions and the renormalization group. CRC Press, 2018.

[14] Robert S Strichartz. Self-similarity on nilpotent lie groups. Contemporary Mathematics, 140:123-123, 1992.

[15] A. K. Chopra. Dynamics of Structures. Prentice Hall, 4th edition, 2011.

[16] AS Veletsos and AH Younan. Dynamic soil pressures on rigid vertical walls. Earthquake engineering \& structural dynamics, 23(3):275-301, 1994.

[17] John H Wood. Earthquake-induced soil pressures on structures. 1973.

[18] Carl M Bender and Steven A Orszag. Advanced mathematical methods for scientists and engineers I: Asymptotic methods and perturbation theory. Springer Science \& Business Media, 2013.

[19] Norman Ricker. The computation of output disturbances from amplifiers for true wavelet inputs. GEOPHYSICS, 10(2):207-220, 1945.

[20] K.F. Graff. Wave motion in elastic solids. Dover, 1975.

[21] Anestis Veletsos and Jethro Meek. Dynamic behaviour of building-foundation systems. Earthquake Engineering \& Structural Dynamics, 3(2):121-138, 1974. 
[22] Bielak Jacobo. Dynamic behaviour of structures with embedded foundations. Earthquake Engineering \& Structural Dynamics, 3(3):259-274, 1975.

[23] K.J. Bathe. Finite Element Procedures. Prentice-Hall International Series in. Prentice Hall, 1996.

[24] T.J.R. Hughes. The Finite Element Method: Linear Static and Dynamic Finite Element Analysis. Dover Civil and Mechanical Engineering. Dover Publications, 2000.

[25] J. P. Wolf. Dynamic soil-structure interaction. Upper Saddle, N.J.: Prentice-Hall., 1985.

[26] J. P. WOLF and C. SONG. To radiate or not to radiate. Earthquake Engineering \& Structural Dynamics, 25(12):1421-1432.

[27] J. Lysmer and R.L. Kuhlemeyer. Finite Dynamic Model for Infinite Media. Reprint (University of California). Department of Civil Engineering, University of California, Institute of Transportation and Traffic Engineering, Soil Mechanics Laboratory, 1969.

[28] Dominic Assimaki. Topography effects in the 1999 Athens earthquake: engineering issues in seismology. $\mathrm{PhD}$ thesis, Massachusetts Institute of Technology. Dept. of Civil and Environmental Engineering., 2004. 